\title{
Systemic lupus erythematosus complicated by tricuspid stenosis and regurgitation: successful treatment by valve transplantation
}

\author{
Diane E Ames, Ronald A Asherson, John D Coltart, Vassilios Vassilikos, \\ J K Lloyd Jones, Graham R V Hughes
}

\begin{abstract}
Clinical tricuspid stenosis has not previously been reported in patients with systemic lupus erythematosus (SLE). A 25 year old woman with active SLE presented with signs of severe right ventricular failure. Cardiac catheterisation confirmed the diagnosis of tricuspid stenosis and regurgitation together with mitral regurgitation. This patient underwent successful tricuspid and mitral valve replacement.
\end{abstract}

It is well established from necropsy studies that cardiac manifestations of systemic lupus erythematosus (SLE) may involve the endocardium, myocardium, pericardium, heart valves, and coronary vessels. ${ }^{1}$ The endocardial involvement described by Libman and Sacks ${ }^{2}$ can be seen in $13-50 \%$ of necropsies, with pericarditis as the most common cardiovascular manifestation. However, only $25 \%$ of such patients show clinical symptoms. With the increasing use of sophisticated methods of diagnosis such as continuous wave Doppler analysis in the routine screening of large numbers of patients with SLE to detect valvular abnormalities, endocardial lesions are becoming increasingly reported in association with this condition. We report here a 25 year old woman with active SLE presenting with signs of severe right ventricular failure, who was subsequently found to have tricuspid stenosis and regurgitation together with mitral regurgitation. This patient underwent successful tricuspid and mitral valve replacements.

Department of

St Thomas's Hospital, London SE1 7EH,

United Kingdom

D E Ames

R A Asherson

G R V Hughes

Department of

Cardiology,

St Thomas's Hospital,

London SE1 7EH,

United Kingdom

J D Coltart

V Vassilikos

Harlow Wood Hospital, Nottingham Road,

Nr Mansfield,

Notts, United Kingdom

J K Lloyd Jones

Correspondence to:

Dr Ronald A Asherson,

Division of Rheumatology and

Connective Tissue Diseases,

The Roosevelt/St Luke's

Hospital Center,

New York 10019, NY, USA

Accepted for publication

8 January 1991 to double stranded DNA. A diagnosis of SLE was made and treatment with tranquillisers and oral prednisolone, to which she had a good symptomatic response, was begun.

Other than occasional pleuritic pains and arthralgias, she remained generally well until September 1988, when she had a 'flare' of SLE; she had, meanwhile, also developed Raynaud's phenomenon. Treatment with azathioprine was begun at this time.

In March 1989 the patient had a further acute psychotic episode and recurrent pleuritic pains and polyarthralgias. On admission to hospital she refused to have a physical examination. She was again treated with tranquillisers, and pulsed methylprednisolone and cyclophosphamide were given intravenously. By August 1989 she had received 13 pulses of cyclophosphamide without clinical improvement. This treatment was discontinued because she developed a severe thrombocytopenia $\left(20 \times 10^{9} / 1\right)$. At the same time, ankle oedema and abdominal swelling, which had not been noted previously, were seen.

In September 1989 the patient was referred to the Lupus Clinic at St Thomas's Hospital with deteriorating renal function. Her main symptoms were breathlessness on exertion, an inability to lie flat, and swelling of her legs and abdomen. She was unwell, pale, withdrawn, and dyspnoeic at rest. Florid nail fold infarcts and livedo reticularis of her hands and legs were seen. She had signs of severe right sided heart failure with sinus tachycardia $(130 / \mathrm{min})$, a jugular venous pressure of $10 \mathrm{~cm}$, a third heart sound, and a loud (grade 4/6) pansystolic murmur at the apex. Her blood pressure on admission was $120 / 75 \mathrm{~mm} \mathrm{Hg}$. She had an enlarged tender liver, gross abdominal ascites and pitting oedema of both legs and abdomen up to the anterior chest wall.

Investigations showed a normochromic normocytic anaemia, once again with a haemoglobin of $76 \mathrm{~g} / \mathrm{l}$. Urea and creatinine levels were raised at 16.3 and $141 \mathrm{mmol} / \mathrm{l}$, respectively. The 24 hour urinary protein and creatinine clearance were within normal limits and there were no urinary casts. A chest $x$ ray showed small lung fields but no significant left ventricular failure. Abdominal ultrasound confirmed free fluid in the peritoneal cavity and showed good blood flow in the inferior vena cava and renal veins with normal sized kidneys. Respiratory function tests were compatible with severe restrictive lung disease. A ventilation/perfusion scan showed no mismatched defects. An electrocardiogram confirmed the sinus tachycardia, axis $+15^{\circ}$ with $T$ wave inversion in the inferior and lateral leads. An echocardiogram showed moderate mitral and tricuspid regurgitation and an enlarged left atrium $(5 \cdot 2 \mathrm{~cm})$.

The left ventricular function was normal. There was thickening of the leaflets of the 
mitral and tricuspid valves and, significantly, a peak gradient of $7 \mathrm{~mm} \mathrm{Hg}$ across the tricuspid valve. Her lupus serology remained active with a DNA binding of $88 \%$, low complement levels (C4 $0.08(0.16-0.45))$ and an erythrocyte sedimentation rate of $62 \mathrm{~mm} / \mathrm{h}$. The antibodies to cardiolipin and lupus anticoagulant tests were negative.

Initially her central venous pressure was monitored and she was treated with low doses of diuretics. She subsequently developed a Staphylococcus aureus septicaemia which responded to flucloxacillin. Inotropic support became necessary at this time to maintain blood pressure and renal output. She subsequently became dependent on dopamine. Cardiac catheterisation was performed. This confirmed good left ventricular function with an ejection fraction of $68 \%$, a normal aortic valve, and grade III mitral regurgitation. Grade III tricuspid regurgitation was also seen with an end diastolic gradient across the valve of $7 \mathrm{~mm} \mathrm{Hg}$. She was referred for urgent surgery.

At surgery, the patient's heart was densely adherent to the pericardium with features of an extremely active pericarditis with constriction. The posterior mitral valve leaflet appeared destroyed with subvalvular debris. There was fusion of the anterior cusps to the septum and septal and lateral leaflets of the tricuspid valve, with subvalvular thrombus. A prosthetic (Cardiomedics $27 \mathrm{~mm}$ ) valve was inserted into the mitral position, and a porcine xenograft replaced the tricuspid valve. Five litres of serum were haemofiltered during surgery.

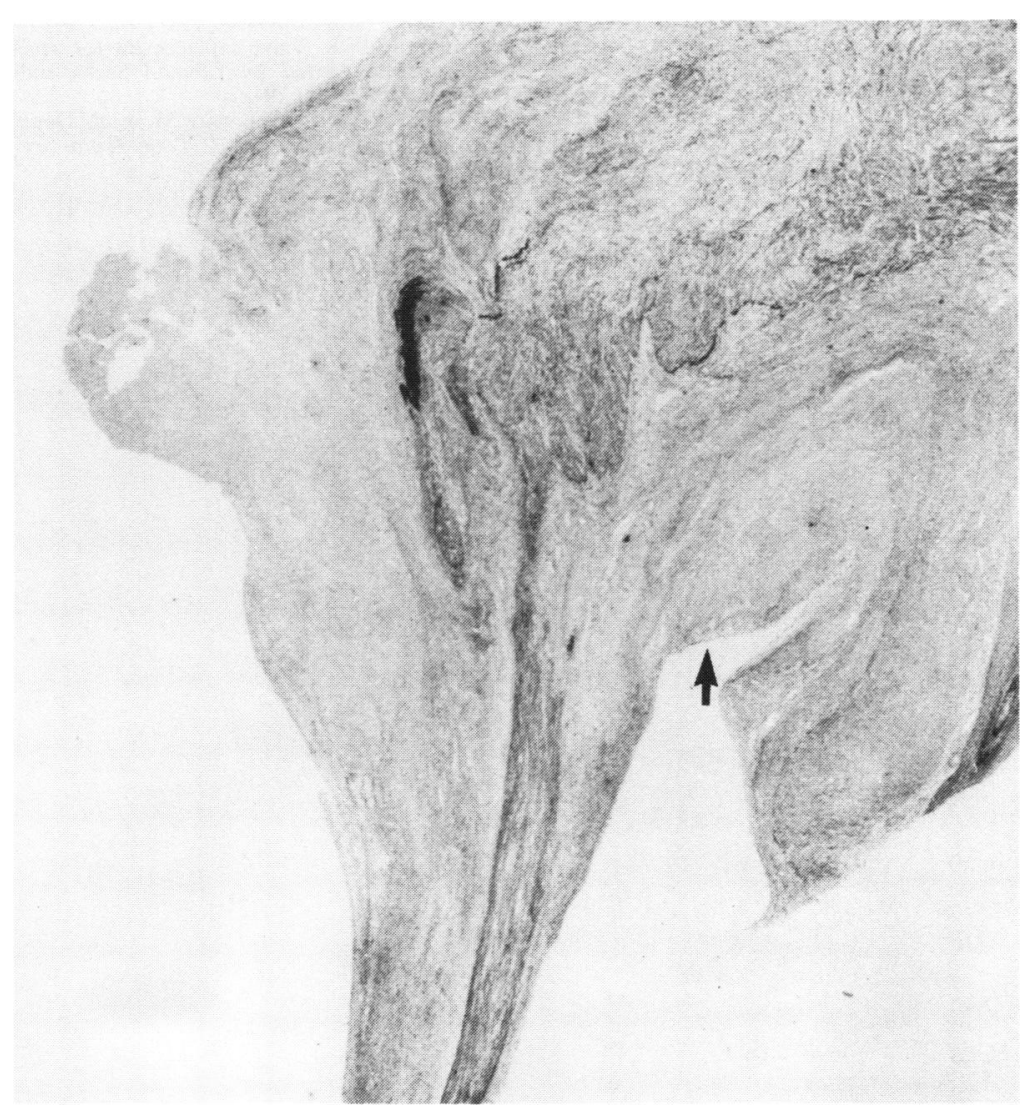

Figure 1 View of the posterior mitral valve leaflet at low power showing the under surface of the leaflet to be filled with collagen tissue (arrow). The chordae tendinea are also involved in the disease process. The surface of the valve leaflet is covered with vascular connective tissue and organising thrombus.

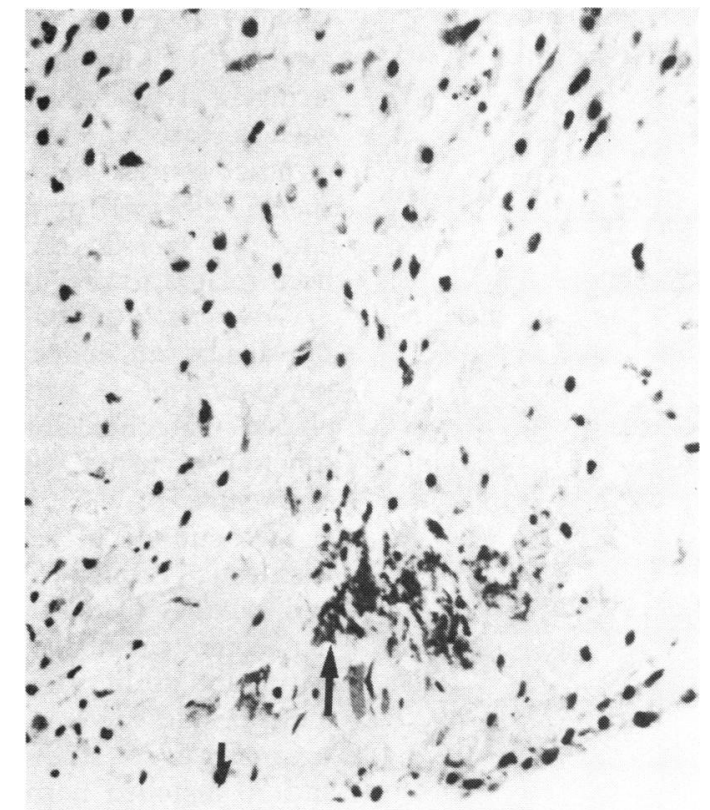

Figure 2 View of the vascular connective tissue filling the gap beneath the posterior mitral valve leaflet (high power). The black areas indicate a focus of fibrinoid necrosis (arrow). No LE cells are seen.

The histology of the valves was consistent with Libman Sacks endocarditis (figs 1 and 2). The mitral valve leaflets were preserved. The anterior leaflet was covered with vascular fibroelastic tissue, as was the adjacent endocardium. The angle under the posterior leaflet was filled with collagen. There were no verrucae. The tricuspid valve was covered with the same vascular fibroelastic tissue and the holding face was covered with thrombus, which also enveloped the chordae and the interatrial septum. Recovery after surgery was unremarkable and she was discharged 10 days later while receiving warfarin, steroids, azathioprine, and a diuretic. At follow up, three months later, she remains well, requiring no change in cardiac treatment, although her lupus serology remains active.

\section{Discussion}

Studies from several centres have shown a higher incidence of endocardial involvement in patients with SLE than previously supposed, and there has also been much interest in the relationship of the antibodies to phospholipids with valve lesions in patients with lupus. Thrombotic endocardial cardiac lesions certainly occur in this context and are often associated with thromboembolic phenomena, particularly to the brain ${ }^{34}$ and coronary vessels. ${ }^{56}$ Although these antibodies were not present in this patient, the mitral valve showed organised thrombosis, indicating that patients other than those with antibodies to phospholipids are at risk of this complication.

Straaton $e t a l^{7}$ reviewed an existing database of 420 patients with SLE collected between 1975 and 1987. They included only those patients with haemodynamically significant 
valvular heart disease for whom tissue was available. Fourteen patients fulfilled these stringent criteria. Six of the 14 had lupus related valve disease, and eight had valve involvement of other causes, such as rheumatic fever, nonspecific fibrosis, or posterior wall infarctions. The non-specific fibrotic lesions might have been related to previous lupus vasculitis.

Galve $e t a l^{8}$ studied echocardiographically the prevalence of endocardial involvement in a population of 74 patients with SLE and concluded that clinically important valvular dysfunction occurred relatively frequently in $18 \%$ of patients.

Two subsets of lesions were identifiable, a verrucous (Libman Sacks endocarditis) subset and valvular thickening and dysfunction causing haemodynamic deterioration. Lesions of the mitral and aortic valves were noted, but not of the tricuspid valve.

Crozier $e t a l^{9}$ studied 50 patients with lupus and 50 matched normal controls. They found that $46 \%$ of the patients with lupus showed mitral regurgitation, whereas $9 \%$ of the controls showed mitral valve prolapse. Aortic incompetence was only seen in $4 \%$ of the patients and not in the controls. Valvular stenosis was absent.

Mandell $^{10}$ reviewed 20 haemodynamically significant valvular lesions reported in patients with SLE and found that aortic regurgitation, mitral regurgitation, mitral stenosis, and mixed mitral lesions were common, with tricuspid regurgitation without stenosis present in only one patient.

Libman and Sacks ${ }^{2}$ found tricuspid valve involvement in necropsy studies; Laufer et $a l^{11}$ described tricuspid valve replacement for severe tricuspid regurgitation in a 9 year old patient.

A survey of reported work has shown that 22 patients with SLE have undergone valve replacement. Three of these have had multiple replacements, SLE being present in most patients for 7-10 years; the mitral valve was the most frequently affected. ${ }^{12}$ Our patient is therefore only the fourth with SLE to undergo multiple replacement. She had pericarditis, found during surgery, mitral regurgitation, and severe tricuspid stenosis with regurgitation, which required two valve replacements for disease attributable to lupus endocarditis. This combination, we believe, has not been reported previously.

Thanks are due to Dr Eckhart Olsen of the National Heart Hospital for advice.

1 Humphreys E M. The cardiac lesions of acute disseminated lupus erythematosus. Ann Intern Med 1948; 28: 12-4.

2 Libman E, Sacks B. A hitherto undescribed form of valvular and mitral endocarditis. Arch Intern Med 1924; 33: 701-37.

3 Doherty N E, Siegel R J. Cardiovascular manifestations of systemic lupus erythematosus. Am Heart $\mathcal{F}$ 1985; 110: 1257-65.

4 Anderson D, Bell D, Lodge M, Grant E. Recurrent cerebral ischaemia and mitral valve vegetation in a patient with antiphospholipid antibodies. $\mathcal{F}$ Rheumatol 1987; 30: 382-8.

5 Murphy J J, Leach I H. Findings of necropsy in the heart of a patient with anticardiolipin syndrome. Br Heart $\mathcal{F} 1989 ; 62$ $61-4$.

6 Brown J H, Doherty C C, Allen D C, Morton P. Fatal cardiac failure due to myocardial microthrombi in systemic cardiac failure due to myocardial microthrombi in

7 Straaton K V, Chatham W W, Reveille J D. Clinically significant valvular heart disease in systemic lupus erythe matosus. Am $\mathcal{F}$ Med 1988; 65: 645-50.

8 Galve E, Candell-Riera J, Pigrau C, Permanyer-Miralda G, Garcia-Del-Castillo H, Soler-Soler J. Prevalence, morphological types and evolution of cardiac valvular disease in systemic lupus erythematosus. $N$ Engl $\mathcal{Y}$ Med 1988; 319: systemic

9 Crozier I, Li E, Milne M, Nicholls M G. Valvular heart disease in systemic lupus erythematosus. $N$ Engl $\mathcal{f}$ Med 1989; 320: 739-40.

10 Mandell B F. Cardiovascular involvement in systemic lupus erythematosus. Semin Arthritis Rheum 1987; 17: 126-41.

11 Laufer J, Frand M, Milo S. Valve replacement for severe tricuspid regurgitation caused by Libman Sacks endotricuspid regurgitation caused by L
carditis. Br Heart $\mathcal{F} 1982 ; 48: 294-7$.

12 Ferraris V A, Hawksley V C, Rabinowitz M, et al. Double valve replacement for lupus valvulitis. Texas Heart Inst $\mathcal{f}$ 1990; 17: 56-60. 